\title{
Patients' perceptions of conservative treatment for a small abdominal aortic aneurysm
}

This article was published in the following Dove Press journal:

Patient Preference and Adherence

\author{
Stephanie M Tomee' \\ Winifred A Gebhardt ${ }^{2}$ \\ Jean-Paul PM de Vries ${ }^{3}$ \\ Victoria C Hamelinck' \\ Jaap F Hamming' \\ Jan HN Lindeman' \\ 'Department of Surgery, Leiden \\ University Medical Center, Leiden, \\ ${ }^{2} \mathrm{Health}$, Medical and Neuropsychology \\ Unit, Institute of Psychology, Leiden \\ University, Leiden, ${ }^{3}$ Department of \\ Vascular Surgery, St Antonius Hospital, \\ Nieuwegein, Leiden, the Netherlands
}

Correspondence: Jan HN Lindeman

Department of Surgery, Leiden University

Medical Center, PO Box 9600, 2300 RC

Leiden, the Netherlands

Tel +3 I 7| 5263968

Email lindeman@lumc.nl
Background: An abdominal aortic aneurysm (AAA) is a progressive, generally symptomless disease that could ultimately result in a fatal rupture. Current guidelines advise conservative follow-up, and preventive surgical repair once the risk of rupture outweighs the cost of repair ( $55 \mathrm{~mm}$ in men). In developed countries, the majority of patients are diagnosed with AAAs less than $55 \mathrm{~mm}$, and so enter a period of conservative surveillance. An important question is how patients perceive and cope with risk of rupture, AAA diagnosis and treatment, and presented AAA information. The goal of this study was to gain insight into patients' perceptions of conservative treatment for a small AAA to increase patient satisfaction.

Methods: We conducted semistructured in-depth interviews and used questionnaires measuring health-related quality of life (RAND 36-Item Health Survey 1.0), illness-perceptions (Illness Perception Questionnaire - Revised), and anxiety and depression (Hospital Anxiety and Depression Scale). Interviews were audio recorded and transcript contents were analyzed based on grounded theory. Mean scores of the questionnaires were compared to (population) reference groups.

Results: This study included ten male patients under surveillance for a small AAA from two hospitals in the Netherlands. Patients expressed no fear for AAA rupture, and also reported low levels of anxiety and depression in both the interviews and the Hospital Anxiety and Depression Scale. The presence of an AAA did not affect their social life or emotional wellbeing. The reported poorer physical health on RAND 36-Item Health Survey 1.0 presumably reflected common presence of comorbidities. Participants stated to that they were content with the frequency and setup of AAA surveillance. However, they generally lacked knowledge about AAA disease and/or treatment options.

Conclusion: Conservative AAA follow-up ensures patients that the risks of AAA disease are limited. The vascular surgeon is the most important source of AAA information to patients, and patients fully rely on their vascular surgeon to take control in AAA treatment.

Keywords: abdominal aortic aneurysm, patient perceptions, patient preference, quality of life, interviews, patient-reported outcome measures

\section{Background}

An abdominal aortic aneurysm (AAA) is a progressive, generally symptomless disease. The natural history of AAA disease is that of gradual dilatation of the abdominal aorta, until a point of insidious rupture. Rupture results in a life-threatening, and often fatal, hemorrhage. ${ }^{1}$ Hence, AAAs are managed by preventive surgical repair. ${ }^{2}$

Although small aneurysms (ie, AAA diameter smaller than $55 \mathrm{~mm}$ in men) do rupture, multiple prospective trials have shown no benefit of surgical elimination of small AAAs. ${ }^{3-6}$ As a result, current guidelines advise a watchful waiting program, and active surgical intervention once the aneurysm reaches the $55 \mathrm{~mm}$ intervention threshold in men. ${ }^{2}$ For women, open repair is indicated at AAA sizes larger than $52 \mathrm{~mm} .^{7}$ Consequently, most patients experience a period of conservative ultrasound follow-up after diagnosis. With the hY $\mathrm{NC}$ and incorporate the Creative Commons Attribution - Non Commercial (unported, v3.0) License (http://creativecommons.org/licenses/by-nc/3.0/). By accessing the work you
hereby accept the Terms. Non-commercial uses of the work are permitted without any further permission from Dove Medical Press Limited, provided the work is properly attributed. For permission for commercial use of this work, please see paragraphs 4.2 and 5 of our Terms (https://www.dovepress.com/terms.php). 
commencement of screening programs, more and more patients are diagnosed with a small AAA. Consequently, the number of patients entering a period of watchful (passive) waiting rises.

There is a remarkable variation in ways the risk of living with a small AAA, either in written information or by health professionals, is presented to patients. In fact, messages vary from "minimal/negligible risk" to "a ticking time bomb." ", A critical question is how patients perceive and cope with the available/presented information, the risk of rupture, and delayed active treatment. The goal of this study was to gain insight into patients' expectations concerning AAA management and to provide health professionals with tools to optimize education and satisfaction of patients under surveillance for a small AAA. To that end, we performed a systematic, explorative collection of patient-reported outcomes by combining in-depth semistructured interviews with validated questionnaires measuring quality of life, ${ }^{10}$ anxiety and depression, ${ }^{11}$ and illness perceptions. ${ }^{12}$

\section{Methods}

This study was performed at the Department of Vascular Surgery from July 2014 till December 2015 in two hospitals in the Netherlands: the Leiden University Medical Center in Leiden and the St Antonius Hospital in Nieuwegein. The study and manuscript were established according to the consolidated criteria for reporting qualitative research. ${ }^{13}$

\section{Ethics approval and consent to participate}

The study protocol was reviewed by the Medical Ethics Committee of the Leiden University Medical Center (C13.094), and the boards of both study centers approved the study. All participants provided written informed consent before participation.

All patient/personal identifiers have been removed or disguised, so the patients/persons described are not identifiable and cannot be identified through the details of the story.

\section{Theoretical framework}

This study was performed within the theoretical framework of grounded theory. ${ }^{14}$ This framework facilitates an explorative approach and applies when qualitative data are scarce and new theories need to be built from data. In this study, this was achieved by combining explorative in-depth interviews in a heterogeneous target group, with validated questionnaires. ${ }^{15,16}$

\section{Participants}

This study includes patients under surveillance for an aneurysm between 35 and $49 \mathrm{~mm}$ in the two participating hospitals. Eligibility screening for participation was done according to the principles of theoretical sampling. ${ }^{14,17}$ This is an established method for creating a heterogeneous sample group with variations in age, educational level, AAA size, time of living with an AAA, and medical history/comorbidity. A total of 12 patients were approached by the vascular surgery staff. Two patients were not interested in participation and declined.

No new information was obtained in the last two interviews performed. Therefore, in accordance with the principles of grounded theory ${ }^{14}$ and theoretical sampling, ${ }^{17}$ it was concluded that data saturation was reached after 8 interviews, and the sample size of 10 interviews was adequate. ${ }^{18,19}$

\section{Interview protocol}

The interview guide aimed at addressing each aspect of living with a diagnosed AAA. All in-depth semistructured interviews were performed by one trained interviewer (SMT), who was not involved in the clinical treatment of AAA patients. The interviews were conducted in Dutch. The questions and responses were translated to English by SMT in collaboration with Dr Roxanne Parslow. The topics addressed in the interview guide are listed in Box 1. The full structure of the interview guide was developed by the authors (primarily SMT in collaboration with WAG, JFH, and JHNL) and is available as Supplementary materials.

Participants were offered the options to have the interview at the hospital or at the participants' home. All individual interviews lasted around 1.5 hours, were audio recorded, and transcribed verbatim. Two of the authors (SMT and VCH) independently created the codes on the basis of two randomly selected interview transcripts. The two authors then established a full set of codes in consensus. These codes were used by SMT to code the remaining interview transcripts. Content analysis of the interview transcript was then performed using the constant comparative method. ${ }^{17}$ According to this method, patient's responses are coded, and then compared within and between interviews. Recurring codes are linked and grouped.

Box I Content of in-depth interviews with participants diagnosed with a small AAA

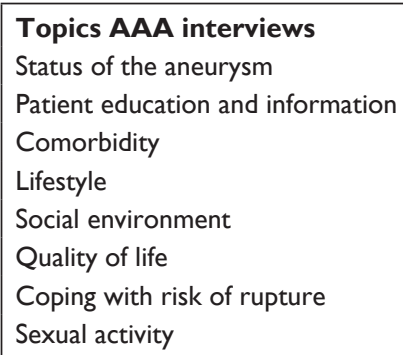

Abbreviation: AAA, abdominal aortic aneurysm. 


\section{Questionnaires}

After the interview, participants received a set of questionnaires and were requested to return them by mail after filling them out at home. The set of questionnaires consisted of health-related quality of life (RAND 36-Item Health Survey 1.0 [SF-36]), ${ }^{10}$ illness perceptions (Illness Perception Questionnaire - Revised [IPQ-R]), ${ }^{12}$ anxiety and depression (Hospital Anxiety and Depression Scale [HADS]), ${ }^{11}$ and a general demographics questionnaire (age, gender, educational level, and [former] profession).

Questionnaires were scored, and total mean scores for all subscales were compared to Dutch population norm scores (SF-36) or reference groups (IPQ-R). Descriptive statistics were performed using SPSS (version 23; IBM Corp., Armonk, NY, USA).

\section{Results}

Participant characteristics are listed in Table 1 . The 10 interviews performed resulted in a total of 16.6 hours of audio recordings. The full set of interview codes is listed in Box 2.

\section{Patients' view and knowledge of AAA disease}

Almost all patients were able to explain the basic concept of aneurysmal disease, usually by the following metaphor: AAA is "like a bicycle tire with a thin spot or bulge on it". However, most patients expressed very limited detailed or accurate knowledge of AAA disease and/or treatment options. For example, one patient was unaware of the fact that an aneurysm can rupture, two patients believed that AAA surgery only slightly reduced the chance of rupture, and two patients believed that an AAA was a narrowing of the blood vessel or flow restriction. In addition, most patients

Table I Sample characteristics of participants with small AAA

\begin{tabular}{ll}
\hline Sample characteristics & $\begin{array}{l}\text { Outcome } \\
(\mathbf{n = 1 0 )}\end{array}$ \\
\hline Gender (\%) & Male (I00\%) \\
Age, years, mean (min-max) & $72.3(63-83)$ \\
AAA diameter, millimeter, mean (min-max) & $42.9(36-48)$ \\
Level of education & 7 lower \\
Marital status & 3 higher \\
& 6 married \\
Time between AAA diagnosis and interview, years, & 3 widower \\
mean (min-max) & I single \\
Anxiety, HADS, mean (SD) ${ }^{a}$ & $5.3(2-I 5)$ \\
Depression, HADS, mean (SD) & \\
\hline
\end{tabular}

Notes: aScales on both anxiety and depression range from 0 to 21 . A score between

0 and 7 per scale excludes the presence of an anxiety disorder or depression.

Abbreviations: AAA, abdominal aortic aneurysm; HADS, Hospital Anxiety and Depression Scale.
Box 2 Interview codes derived from content analysis of ten interview transcripts

\section{Status of the aneurysm}

AAA is incidental finding

Has experienced AAA growth in the past

Is aware of nature and/or risk of AAA disease

Is content with frequency of AAA follow-up

Easily accepts changes in follow-up frequency if necessary

Finds it reassuring that $A A A$ is given attention through follow-up

Does not worry about AAA

Trusts vascular surgeon to do what is best in AAA treatment

Can describe AAA disease in simple terms

Cannot describe AAA disease in simple terms

Patient education and information

Is able to reproduce orally presented AAA information in simple

terms

Does not need extra AAA information

Did not use internet for extra AAA information

AAA information from the internet can cause one to worry

Is satisfied with AAA information provided by the vascular surgeon

Has not received written AAA information during hospital visits

Prefers to hear results directly after completing ultrasound

examination

\section{Comorbidity}

History of cardiovascular disease

History of cancer

History of chronic obstructive pulmonary disease

No physical burden of AAA

Other concomitant diseases are physically more challenging than AAA No mental burden of AAA

Other concomitant diseases are mentally more challenging than AAA

Accepts personal health state

Mobility is most important aspect of health

Lifestyle

Former smoker

AAA diagnosis did not influence lifestyle (with regard to smoking,

alcohol consumption, diet, exercise)

Has a positive view on personal future

Has a positive attitude toward AAA national screening program

Social environment

Does not feel the need to talk about AAA with others

Feels like other people perceive an AAA as more severe than

participant itself

Is content with personal (social) life

Has acquaintance(s) with AAA experience

AAA event in social network did not influence personal view on own

AAA

Quality of life

Quality of life is defined as follows: no restrictions in living life as you want it

Accepts personal limitations from health or older age

Feels no need to make use of facilities provided by an AAA patient

association

Coping with risk of rupture

Is aware that AAA can rupture

Is aware that AAA rupture is often fatal

Does not fear AAA rupture

Has no knowledge of physical symptoms that would accompany a

rupture

Never experienced believing that own AAA had ruptured

Does not perceive AAA as a "ticking time bomb"

Is content with current conservative treatment plan

Does not have accurate knowledge of all AAA treatment options

Sexual activity

AAA has or had no influence on sexual life

Abbreviation: AAA, abdominal aortic aneurysm. 
perceived the $55 \mathrm{~mm}$ intervention threshold as a "critical threshold" beyond which an AAA abruptly becomes dangerous. "I sort of know what it [aneurysm] is. Your aorta is too wide or too narrow. I don't know exactly." [Patient 1, 69 years].

\section{Patients' view on AAA information provided by hospital (staff)}

Despite the apparent lack of accurate information on their AAA, the majority of patients stated to be satisfied with the oral information provided by the vascular surgeon. They expressed no desire for additional AAA information. Patients felt strongly reassured by the surgeon's message that there was no need for immediate surgery, and that the next follow-up would be in another 6 or 12 months. "If they let me go home for another six months after examining it [aneurysm], it can't be that dangerous". [Patient 8, 63 years]. In addition, all patients fully relied on the vascular surgeon to do "what is best for me", and all preferred the surgeon to take the lead in their AAA management.

\section{Fear for AAA rupture}

Almost all participants explicitly stated not to experience any fear for AAA rupture. This is also reflected in low levels of anxiety and depression as measured by the HADS ${ }^{11}$ (Table 1), and low scores on the "consequences" and "emotional representation" subscales of the IPQ-R ${ }^{12}$ (Figure 1). IPQ-R reference scores for laymen perceptions of AIDS, skin cancer, and tuberculosis ${ }^{20}$ are included for comparison.

Explicitly, none of the patients perceived their AAA as a "ticking time bomb". "To state that you walk around with a ticking time bomb, well I don't perceive it that way." [Patient 5, 75 years].

\section{Physical and mental burden of AAA disease}

All but two participants experienced various comorbidities such as cardiovascular disease, cancer, and COPD. None of the patients experienced any AAA-related symptoms. Regardless of the presence of comorbidity, all patients considered the physical and mental burden of an aneurysm to be minimal. On a scale ranging from 0 (very bad) to 10 (excellent), participants graded their general health with a mean score (SD) of 6.9 (1.3), and their quality of life with a mean score (SD) of 7.7 (0.9). All patients expressed a positive attitude toward their health. In addition, they enjoyed their lives despite experiencing physical discomfort from comorbidities and older age.

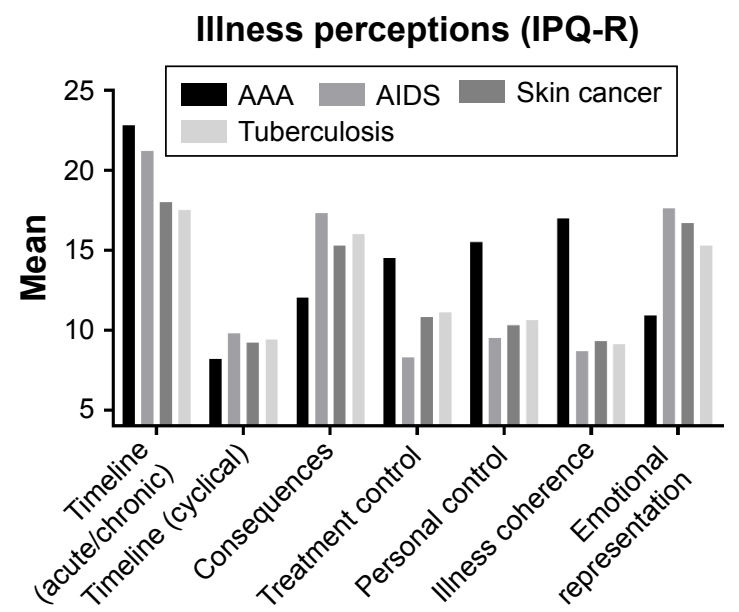

Figure I Mean scores of AAA patients $(n=10)$ on the illness perceptions questionnaire (IPQ-R), compared to laymen illness perceptions (mean scores) of AIDS ( $n=379)$, skin cancer $(n=391)$, and tuberculosis $(n=379)$.

Notes: Higher scores on "Timeline (acute/chronic)" is more belief in chronicity of the disease, scale ranges from 6 to 30. Higher scores on "Timeline (cyclical)" is more belief in variability of symptoms, scale ranges from 4 to 20 . Higher scores on "Consequences" is more belief in serious consequences of the disease, scale ranges from 6 to 30 . Higher scores on "Treatment control" is more belief in effect of treatment for the disease, scale ranges from 5 to 25 . Higher scores on "Personal control" is more belief in personal control over the disease, scale ranges from 6 to 30. Higher scores on "Illness coherence" is more effectiveness of patients' disease model for explaining the disease/symptoms, scale ranges from 5 to 25 . Higher scores on "Emotional representation" is more negative feelings toward the disease, scale ranges from 6 to 30. The data used to create this image is from reference groups and therefore necessary for comparison. ${ }^{20}$

Abbreviations: AAA, abdominal aortic aneurysm; IPQ-R, Illness Perception Questionnaire - Revised.

SF-36 scores showed that AAA patients experience poorer physical health states than the Dutch reference population (Figure 2). ${ }^{10}$ Scores on the social functioning and emotional well-being subscales of the SF-36 were similar for AAA patients and the reference population. "Well I still rate my own

Health-related quality of life (SF-36)

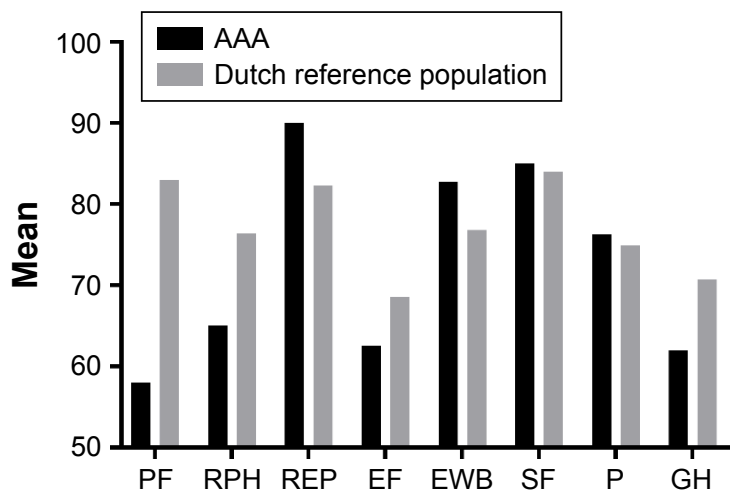

Figure 2 Mean scores of AAA patients $(n=10)$ and Dutch general reference population $(n=1,742)$ on the Shortform-36 (SF-36) measuring health-related quality of life. Notes: Scales range from 0 to 100 . Higher scores indicate a more favorable health state. The data used to create this image is from reference groups, and therefore necessary for comparison. ${ }^{10}$

Abbreviations: AAA, abdominal aortic aneurysm; PF, physical functioning; $\mathrm{RPH}$, role limitations due to physical health; REP, role limitations due to emotional problems; EF, energy/fatigue; EWB, emotional well-being; SF, social functioning; $\mathrm{P}$, pain; $\mathrm{GH}$, general health. 
health with a 7." [Patient 4, 69 years, living with peripheral arterial disease (Fontaine II) and metastasized prostatic cancer].

\section{Patients' view on AAA surveillance}

All participants were content with the (frequency and setup of) periodical ultrasound follow-up. However, situations in which patients had to wait a few days or weeks to hear the results of AAA examination were experienced as highly frustrating. "Sometimes it takes 14 days after the ultrasound before you hear the result from the doctor. During those 14 days, I am a bit anxious. Those 14 days are difficult." [Patient 10, 77 years].

\section{Discussion}

This study shows that, in contrast to widely held beliefs, ${ }^{8,21-23}$ patients under surveillance for a small AAA do not suffer from having an AAA, and do not perceive their AAA as a "ticking time bomb." In fact, patients experience the conservative nature of AAA surveillance as reassuring. However, most patients perceive the $55 \mathrm{~mm}$ intervention threshold as an abrupt transition, upon which the AAA suddenly becomes dangerous.

This study applies a combination of in-depth, semistructured interviews and questionnaires, ${ }^{15,16}$ a validated approach for an unbiased and systematic exploration of patients' perceptions. This explorative approach is well established in social ${ }^{15,24,25}$ and environmenta ${ }^{26}$ sciences, but is relatively new in the field of medicine. Data are acquired through both qualitative (semistructured in-depth interviews) and quantitative methods (questionnaires). The advantage of this approach is that it allows for a more comprehensive, unbiased, and systematic exploration of patientreported outcomes than conventional, exclusive qualitative or quantitative methods. The purpose of this study was to increase understanding of the experiences and expectations of patients living with a small AAA. Results allow vascular health professionals to optimize patient counseling and patient satisfaction.

One of the most notable findings in the study is that all patients are very content with conservative treatment. All participants in the study are under surveillance by different vascular surgeons in different hospitals. This implies that this is a general, doctor- and hospital-independent finding. Patients indicated that the conservative nature of surveillance reassured them that the risks of having a small AAA are limited.

This study showed that patients often have dysfunctional or incorrect beliefs about AAA disease or treatment options, as such it is recommended that vascular specialists check patient beliefs during consultations. A specific point of attention is the apparent need for reassuring information once AAA diameter approaches the $50-55 \mathrm{~mm}$ intervention threshold. Upcoming questions or concerns should be regularly evaluated with patients.

Remarkably, findings of our study contrast with results from previous studies on the impact of having an AAA. An earlier report by Lindholt et $\mathrm{al}^{23}$ stated that a screening detected small AAA (progressively) impairs quality of life in conservatively treated patients. This conclusion was based on the results of a health-related quality-of-life questionnaire (ScreenQL). An important notion is that most quality-of-life questionnaires measure an "overall quality-of-life state" and do not discriminate between AAA-related health issues or other health issues. In fact, results of the ScreenQL questionnaire performed as part of this study also show that AAA patients have worse general health compared to the general population due to the presence of comorbidities. ${ }^{23}$ Consequently, the conclusion that "AAA progressively impairs quality of life" is therefore not substantiated.

Another qualitative study by Petterson et $\mathrm{al}^{27}$ reported feelings of anxiety, uncertainty about physical symptoms, and fear for AAA growth in AAA patients. These observations suggest a major impact of AAA diagnosis and delayed surgery in patients under surveillance. We have no obvious explanation for the discrepancies between our findings and those of Petterson et al. ${ }^{27}$ Possible explanations include not only the cultural differences between Sweden and the Netherlands in ways patients and doctors perceive AAA disease, but also the fundamental differences in methodology between both studies. Petterson et a ${ }^{27}$ used an explorative "life-world" approach in which insights to a patient's life world is gained through storytelling. However, lack of structure in this approach interferes with consistency and reproducibility of the interviews. Moreover, the results by Petterson et $\mathrm{al}^{27}$ should be reviewed in the context of a nationwide AAA-screening program. The potential risks of having an (undiagnosed) AAA are often emphasized in AAA-screening invitations as a means of motivating people to participate in the screening. ${ }^{9}$ It is very likely that this type of patient information could have a long-term and profound influence on patients' perceptions of AAA disease. ${ }^{27}$

This study has a few points of consideration. First of all, our findings could be specific for "Dutch culture" and can, therefore, not automatically be translated to AAA patients in 
other countries (with or without AAA screening). Replicating this study in other countries could identify any culturespecific differences in coping with AAA disease.

A second limitation is the fact that female AAA patients are not represented in this study. To the best of our knowledge, there are no reports of qualitative studies with female AAA patients available. However, other studies identified gender differences in illness perceptions of allergic rhinitis ${ }^{28}$ and osteoporosis, ${ }^{29}$ indicating that female patients perceive diseases differently than men. As current guidelines emphasize the need for gender-specific AAA treatment and suggest considering AAA repair at lower diameters in women, ${ }^{2}$ there is a great need for involving female patients in patientreported outcome studies.

Finally, our sample is susceptible to selection bias, as this is inherent to most qualitative interview studies. We have attempted to minimize selection bias by creating a heterogeneous sample through theoretical sampling. Nevertheless, this remains a point of consideration when generalizing the findings from this study to the general patient population.

\section{Conclusion}

This study shows that patients are very content with frequency and setup of AAA surveillance, which ensures patients the risks of a small AAA are limited. Patients regard the vascular surgeon as the most important source of AAA information.

Concerning surveillance, most patients are content with periodical ultrasound, although having to wait for the results can evoke frustration in patients. Combining the ultrasound examination and consultation with the vascular surgeon on the same day in outpatient clinical practice could increase patient satisfaction. At last, increasing the frequency of AAA surveillance does not necessarily result in more reassurance in patients. Of note, these findings are restricted to men, as studies in female AAA patients are needed.

\section{Acknowledgments}

We thank Dr Roxanne Parslow for providing language help with the English translation of the interview guide. In addition, we thank all participants in this study for their cooperation.

\section{Author contributions}

SMT, WAG, JFH, and JHNL contributed toward the study concept and design. SMT, JPPMV, and JFH dealt with reaching out to patients. SMT was responsible for acquisition of data. SMT, WAG, VCH, JFH, and JHNL conducted statistical analysis and interpretation of data. SMT, WAG, JFH, and JHNL drafted the manuscript. SMT, WAG, JPPMV, VCH, JFH, and JHNL revised the manuscript critically for intellectual content. JPPMV, JFH, and JHNL supervised the study. All authors contributed toward data analysis, drafting and critically revising the paper, gave final approval of the version to be published, and agree to be accountable for all aspects of the work.

\section{Disclosure}

The authors report no conflicts of interest in this work.

\section{References}

1. Thompson SG, Brown LC, Sweeting MJ, et al. Systematic review and meta-analysis of the growth and rupture rates of small abdominal aortic aneurysms: implications for surveillance intervals and their costeffectiveness. Health Technol Assess. 2013;17:1-118.

2. Moll FL, Powell JT, Fraedrich G, et al. Management of abdominal aortic aneurysms clinical practice guidelines of the European society for vascular surgery. Eur J Vasc Endovasc Surg. 2011;41(Suppl 1):S1-S58.

3. Mortality results for randomised controlled trial of early elective surgery or ultrasonographic surveillance for small abdominal aortic aneurysms. The UK Small Aneurysm Trial Participants. Lancet. 1998; 352:1649-1655.

4. Lederle FA, Wilson SE, Johnson GR, et al. Immediate repair compared with surveillance of small abdominal aortic aneurysms. $N$ Engl J Med. 2002;346:1437-1444.

5. Cao P, De Rango P, Verzini F, et al. Comparison of surveillance versus aortic endografting for small aneurysm repair (CAESAR): results from a randomised trial. Eur J Vasc Endovasc Surg. 2011;41:13-25.

6. Ouriel K, Clair DG, Kent KC, Zarins CK; Positive Impact of Endovascular Options for treating Aneurysms Early I. Endovascular repair compared with surveillance for patients with small abdominal aortic aneurysms. J Vasc Surg. 2010;51:1081-1087.

7. Tomee SM, Lijftogt N, Vahl AC, Hamming JF, Lindeman JHN. A registry-based rationale for discrete intervention thresholds for open and endovascular elective abdominal aortic aneurysm repair in female patients. J Vasc Surg. In press 2017.

8. Aoki H. Taking control of the time bomb in abdominal aortic aneurysm. Circ J. 2016;80:314-315.

9. NHS. Abdominal aortic aneurysm (AAA) screening: Patient information; 2014 [cited January 6, 2017]. Available from: www.nhs.uk/aaa

10. Aaronson NK, Muller M, Cohen PDA, et al. Translation, validation, and norming of the Dutch language version of the SF-36 Health Survey in community and chronic disease populations. J Clin Epidemiol. 1998;51:1055-1068.

11. Zigmond AS, Snaith RP. The hospital anxiety and depression scale. Acta psychiatr scand. 1983;67:361-370.

12. Moss-Morris R, Weinman J, Petrie KJ, Horne R, Cameron LD, Buick D. The revised Illness Perception Questionnaire (IPQ-R). Psychol Health. 2002;17:1-16.

13. Tong A, Sainsbury P, Craig J. Consolidated criteria for reporting qualitative research (COREQ): a 32-item checklist for interviews and focus groups. Int J Qual Health Care. 2007;19:349-357.

14. Glaser BG, Strauss AL. The Discovery of Grounded Theory: Strategies for Qualitative Research. Chicago: Aldine Publishers; 1967.

15. Tashakkori A, Teddlie C. Handbook of Mixed Methods in Social and Behavioral Research. 2nd ed. Thousand Oaks: Sage Publications; 2010.

16. Johnson RB, Onwuegbuzie AJ, Turner LA. Toward a definition of mixed methods research. J Mix Methods Res. 2007;1:112-133.

17. Boeije H. A purposeful approach to the constant comparative method in the analysis of qualitative interviews. Qual Quant. 2002;36:391-409. 
18. Morse JM. The significance of saturation. Qual Health Res. 1995;5: 147-149.

19. Morse JM. Determining sample size. Qual Health Res. 2000;10:3-5.

20. Figueiras MJ, Alves NC. Lay perceptions of serious illnesses: an adapted version of the Revised Illness Perception Questionnaire (IPQ-R) for healthy people. Psychol Health. 2007;22:143-158.

21. Hansson A, Brodersen J, Reventlow S, Pettersson M. Opening Pandora's box: the experiences of having an asymptomatic aortic aneurysm under surveillance. Health Risk Soc. 2012;14:341-359.

22. Howell CM, Rabener MJ. Abdominal aortic aneurysm: a ticking time bomb. J Am Acad Physician Assist. 2016;29:32-36.

23. Lindholt JS, Vammen S, Fasting H, Henneberg EW. Psychological consequences of screening for abdominal aortic aneurysm and conservative treatment of small abdominal aortic aneurysms. Eur J Vasc Endovasc Surg. 2000;20:79-83.

24. Greene JC. Mixed Methods in Social Inquiry. San Francisco: JosseyBass; 2007.
25. Deasy C, Coughlan B, Pironom J, Jourdan D, Mannix-McNamara P. Psychological distress and coping amongst higher education students: a mixed method enquiry. PLoS One. 2014;9:e115193.

26. Free CM, Jensen OP, Mendsaikhan B. A Mixed-method approach for quantifying illegal fishing and its impact on an endangered fish species. PLoS One. 2015;10:e 0143960.

27. Pettersson M, Bergbom I. To be under control: a qualitative study of patients' experiences living with the diagnosis of abdominal aortic aneurysm. J Cardiovasc Nurs. 2013;28:387-395.

28. Pesut D, Raskovic S, Tomic-Spiric V, et al. Gender differences revealed by the brief Illness Perception Questionnaire in allergic rhinitis. Clin Respir J. 2014;8:364-368.

29. Edelstein OE, Werner P, Dresner-Pollak R, et al. Illness perceptions among osteoporotic men and women: correlates and gender differences. J Mens Health. 2012;9:168-175. 


\section{Supplementary material}

The interviews were conducted in Dutch. The questions and responses were translated to English by SMT in collaboration with Dr Roxanne Parslow.

\section{Interview guide on small abdominal aortic aneurysm (translated from Dutch)}

Introduction: "The subject of this interview will focus on you as a patient, living with the condition, abdominal aortic aneurysm. The purpose of this study is to use the information obtained from this interview to improve the treatment, care, or services for patients with an aneurysm. This conversation takes place in a confidential setting, in which you are free to say whatever you would like to say. We would like to know about your experiences, how you feel, and what you think, so do not hesitate to respond with your honest opinion. The responses you give in this interview are anonymous and cannot be traced back to you as a person. It is also important that you know that the responses you give will have no influence on your treatment and will not be heard by (one of) your doctor(s).

Some of the subjects in this interview might be challenging or upsetting for you. If you experience this, or you find this difficult, please let us know during the interview. If you do find a question challenging, we hope you will still try to answer, as those questions are probably the ones we do not have much knowledge about. However, you do not have to answer every question. If a question is not clear to you, please ask us to clarify this.

Do you have any questions or comments you would like to address before we begin?"

(For the interviewer: during the interview, use the follow-up question "Could you tell me more about this?" if additional information is needed.)

Open with:

1. Could you start by telling me something about yourself?

2. If you would have to explain to your neighbor what an aneurysm is, how would you explain this?

\section{Status of the aneurysm}

Introduction of the subject: "I would like to start with a few short, general questions about the diagnosis and treatment of your aneurysm."

1. When was the aneurysm diagnosed in your case?

2. How was the aneurysm discovered?

3. What was the size of the aneurysm at diagnosis?

4. Has the aneurysm grown in size since the diagnosis?

5. How often do you receive surveillance for the aneurysm? Is this by ultrasound or $\mathrm{CT}$ ?
6. How often would you like to receive surveillance for the aneurysm? More or less frequently? Why?

7. How has the aneurysm surveillance been?

\section{Patient education and information}

"With the following questions, I would like to know more about the information about aneurysms that you have received in this hospital and what you think about it. Could you tell me more about this?"

Hospital

8. Did you receive information in this hospital about an aneurysm at diagnosis? (This could be written information or oral information from a doctor.)

9. Did you receive information in this hospital about an aneurysm during your surveillance period?

10. If yes, what kind of information was this? (written/oral?)

11. Was this information sufficient in your opinion?

- After receiving and/or reading it, did you learn everything you wanted to know?

12. What did the information give you?

- Did it provide reassurance or did it cause more worry?

13. Did the information change your view of an aneurysm?

- If yes, how did it change your view?

- If no, why did it not change your view?

14. Do you feel like you can say or ask anything during a consultation with the vascular surgeon?

- If yes, did you say or ask anything during a consultation with the vascular surgeon?

- If no, why not?

Internet

15. Did you search the internet for information about aneurysms?

- If yes, did you find what you were looking for?

- If no, why not?

16. Was that information sufficient in your opinion?

- If no, what did you still want to know?

17. What did the information give you?

- Did it provide reassurance or did it cause more worry?

18. Did the information change your view of an aneurysm?

- If yes, how did it change your view?

- If no, why did it not change your view?

\section{Comorbidity}

"During the following questions, I would like to ask more about whether you have any other diseases."

19. Do you have or had any other medical diseases?

- If yes, what do you have, and can you tell me more about it? 
20. Do you have any other physical symptoms?

- If yes, can you tell me more about it?

21. Could you make a list of all the medical diseases or physical symptoms you have and rank them based on their physical burden, where number 1 has the highest physical burden and rank the rest in descending order? With physical burden, we mean experiencing physical discomfort or limitation.

22. Could you make a list of all the medical diseases or physical symptoms you have and rank them based on their mental burden, where number 1 has the highest mental burden and rank the rest in descending order? With mental burden, we mean experiencing worries or emotions or being mentally occupied by it.

23. If you were asked to grade your own health with a grade between 1 and 10, where 10 is excellent and 1 is very bad, what would that grade be?

- How was your health in the past, for instance, 5 years ago? What would that grade be then?

24. When reviewing your current health, what would be needed to raise that grade?

25. What does "health" mean to you? Which aspects play a role when reviewing your own health?

\section{Lifestyle}

"With these next questions, I would like to ask more about your lifestyle."

26. Do you smoke?

- If yes, do you smoke more than one pack per week? How much exactly?

- If yes, have you ever smoked more than one pack per week? How much was this and for how long?

- If no, have you ever smoked?

- Has your smoking habit changed after being diagnosed with an aneurysm? $\rightarrow$ If yes, why and how?

27. Do you drink alcohol?

- If yes, do you drink more than two alcoholic beverages per day?

- Has your alcohol consumption changed after being diagnosed with an aneurysm? $\rightarrow$ If yes, why and how?

28. Do you sleep more than 6 hours per night?

- Do you sleep well?

- If no, why not?

- Has your sleeping habit or quality changed after being diagnosed with an aneurysm? $\rightarrow$ If yes, why and how?

29. Do you get exercise or do you do sports?

- If no, why not?
- If yes, what type of exercise/sports per week? Has this changed after being diagnosed with an aneurysm? $\rightarrow$ If yes, why and how?

30. What do you generally eat during a normal day?

- Has your eating pattern changed after being diagnosed with an aneurysm? $\rightarrow$ If yes, why and how?

31. How do you see your future and in what way has this changed since you found out you have an aneurysm?

32. In hindsight, would you rather not know that you have an aneurysm?

33. In the Netherlands, there is an ongoing debate about starting a national AAA screening program in which men over 65 years are being screened for an AAA through an abdominal ultrasound. What do you think about a national AAA screening program?

\section{Social environment/social activities}

"In the next questions, I would like to know more about your social life and whether being diagnosed with an aneurysm possibly had any influence on this. Could you tell me more about this?"

34. Could you tell me something about how and with whom you live?

35. Do you have people in your (social) life, you can talk to about personal things if you want to?

- If yes, with who did/do you talk?

36. When was the last time you talked about your aneurysm with anyone?

- What did you discuss?

37. What has talking about your aneurysm with someone else brought you?

- Did it provide reassurance or did it cause more worry?

38. Do you know anyone personally who has had experience with an aneurysm?

- If yes, could you tell me more about this?

39. Did your social life change after being diagnosed with an aneurysm?

- Do you still do the same things you did before, like vacations, hobbies, or visiting friends or family?

- If yes, could you tell me more about this?

\section{Quality of life}

"Current health care is focused on maintaining or improving someone's quality of life. With the following questions, I would like to learn more about what your definition of and view is on your 'quality of life."' (Explain quality of life if necessary as follows: being able to live your life as you want to.)

40. What is your definition of "quality of life?"

- What aspects are important to you when thinking about "quality of life?" 
41. If you were asked to grade your own quality of life with a grade between 1 and 10, where 10 is excellent and 1 is very bad, what would that grade be?

- How was your quality of life in the past, for instance, 5 years ago? What would that grade be then?

42. When reviewing your current quality of life, what would be needed to raise that grade?

43. If you could live a perfect life, what would that look like? And why would that be perfect?

44. If there was some sort of organization of foundation for people with an aneurysm that you could use for information or contacting other people with an aneurysm etc, would you be interested in using that for yourself?

\section{Coping with chance of rupture}

(Introduce only when previously established that the patient is aware of risk of rupture.)

"As you probably know the danger of having an aneurysm is that it can rupture (burst). Although the chances of rupture are very slim, it remains an unpredictable disease. The following question will ask about your view about this and the way you handle the risk of rupture."

45. Do you know what the symptoms are of a ruptured aneurysm? $\rightarrow$ If yes, could you tell me more?

46. What do you think the chance of the aneurysm rupturing is in your case?

47. Do you worry about the aneurysm? About aneurysm rupture? $\rightarrow$ If yes, could you tell me more?

48. Do you worry the aneurysm has ruptured whenever you experience abdominal or back pains or when you feel ill? $\rightarrow$ If yes, could you tell me more?

49. Have you ever thought your aneurysm has ruptured? $\rightarrow$ If yes, could you tell me more?
50. Did you ever consult a doctor because you thought that your aneurysm had ruptured? $\rightarrow$ If yes, could you tell me more?

51. Do you take the aneurysm (and the possibility of rupture) into account when doing things in your daily life?

- Are there any things you do not do anymore because of the aneurysm?

- If yes, could you tell me more?

52. If you could choose, what kind of treatment would you prefer for the aneurysm and why? (Explain treatment options if necessary: no treatment, surveillance, open abdominal surgery, or endovascular aneurysm repair.)

53. Popularly speaking, an aneurysm is often referred to as a "ticking time bomb," how do you feel about this?

\section{Sexual activity}

"We know from previous conversations that having an aneurysm can limit someone in their sexual life, in the form of feeling inhibited, reduced frequency, or less excitement for instance."

54. Have you ever experienced this?

- If yes, could you tell me more?

55. This is the end of the interview. Do you think that we covered all things that matter to you?

- If no, is there anything you would like to add, ask, or tell?

Thank you very much for your cooperation.

--------End of interview--------
Patient Preference and Adherence

\section{Publish your work in this journal}

Patient Preference and Adherence is an international, peer-reviewed, open access journal that focuses on the growing importance of patient preference and adherence throughout the therapeutic continuum. Patient satisfaction, acceptability, quality of life, compliance, persistence and their role in developing new therapeutic modalities and compounds to optimize

\section{Dovepress}

clinical outcomes for existing disease states are major areas of interest for the journal. This journal has been accepted for indexing on PubMed Central. The manuscript management system is completely online and includes a very quick and fair peer-review system, which is all easy to use. Visit http://www dovepress.com/testimonials.php to read real quotes from published authors. 